\title{
Conflicts around a study of Mexican crops
}

\author{
Nature published a paper last year claiming that transgenic DNA had become genetically \\ incorporated into traditional maize in Mexico. A debate ensued ...
}

Sir - Scientific endeavour is based on formation and testing of hypotheses. Very few hypotheses persist unmodified after they are first proposed; most are tested, modified and re-tested, and often refuted. Many journals include a forum for scientists to make technical comments on recent publications and for the original authors to respond, so that readers can evaluate the merit of reported scientific findings.

The cornerstones of a rigorous publication process are the subject editor, who is familiar with the research area of a submitted manuscript, and the independent outside reviewers whose recommendations are solicited by the subject editor. This thorough evaluation ensures, to some extent, the journal's impartiality in publication decisions.

Despite this rigorous process, however, Nature recently published a technical exchange $\mathrm{e}^{1-3}$ accompanied by an editorial note stating: "Nature has concluded that the evidence available is not sufficient to justify the publication of the original paper".

In our view this statement reflects poorly on Nature's editorial policy and review process, and sets a dangerous precedent. Why has Nature refrained from releasing similar editorial retractions of earlier publications later found to be incorrect or open to alternative interpretations? What sets this particular publication apart? If the interpretation of the results proposed by the authors of the original paper ${ }^{4}$ was judged by Nature to be sufficiently erroneous to warrant this editorial statement, why did Nature publish the report in the first place?

By taking sides in such an unambiguous manner, Nature risks losing its impartial and professional status. This is particularly troubling when articles are related to economic or political interests. Nature asks its contributors to provide information regarding conflicts of interest, but does Nature hold itself to the same standards?

\section{Andrew V. Suarez}

Department of Environmental Science, Policy and Management, Division of Insect Biology, University of California, 201 Wellman Hall, Berkeley,

California 94720-3112, USA

Other signatories to this letter:

Mike Benard, Neil D. Tsutsui Department of Evolution and Ecology, Center for Population Biology, University of California, Davis, USA Todd A. Blackledge Department of Entomology, Cornell University, Ithaca, USA

Kirsten Copren, Eli M. Sarnat, Alex L. Wild Department of Entomolog $\gamma$, University of California, Davis, USA

Wayne M. Getz, Philip T. Starks, Kipling Will, Per J. Palsbøll

Department of Environmental Science, Policy and Management,
University of California, Berkeley, USA

Mark E. Hauber Department of Neurobiology and Behavior, Cornell University, USA

Craig Moritz Department of Integrative Biology, University of California, Berkeley, USA

Adam D. Richman Department of Plant Science and Plant Pathology, Montana State University, USA

Sir - The controversy surrounding Quist and Chapela's findings of transgenic introgression in Mexican maize $\mathrm{e}^{1-4}$ is taking place within webs of political and financial influence that compromise the objectivity of their critics.

The eight authors of the two published criticisms $^{1,2}$ of Quist and Chapela's paper ${ }^{4}$ have had all or part of their research funded by the Torrey Mesa Research Institute (TMRI), an offspring of the agricultural biotechnology company Novartis (now Syngenta).

The affiliation of seven of those authors with TMRI is a result of that company's \$25-million 'strategic alliance' with the University of California (UC), Berkeley's College of Natural Resources. Wilhelm Gruissem, formerly of UC, Berkeley and architect of the strategic alliance, whose laboratory is in partnership with TMRI, is the supervisor of the eighth author, Johannes Fütterer. None of the eight authors declares this funding as a competing financial interest in their published contributions.

Such a funding arrangement might be less noteworthy had Chapela and Quist not been leading critics of the strategic alliance and its implications for scientific freedom and balance. Their vocal opposition to the alliance jeopardized a large flow of financial support for the authors of the criticisms.

Compromised positions extend beyond those of these critics. Nature Publishing Group actively integrates its interests with those of companies invested in agricultural and other biotechnology, such as Novartis, AstraZeneca and other 'sponsorship clients', soliciting them to "promote their corporate image by aligning their brand with the highly respected Nature brand" (see http://npg.nature.com). These partnerships seem to us to challenge Nature's ability to provide a neutral forum for scientific debates on agricultural biotechnology.

Nature's editorial note disavowing Quist and Chapela's work in the same issue as the technical exchanges ${ }^{1-3}$ was unorthodox and unnecessary. The usual scientific process of contestation should have been permitted to proceed, using
Quist and Chapela's claims and data alone to repeat, verify or refute their findings. Because of its potential effect on regulatory policy, publication of the technical exchanges and Nature's editorial note immediately before the UNEP Convention on Biological Diversity meeting and discussions of the Cartagena Protocol on Biosafety (where Nature's editorial note was mentioned) further undermines the journal's stance as uncompromised by commercial interests.

In such an environment, it is difficult to imagine fair consideration being given to work that challenges commercially vested interests and the assumptions of reductionist molecular biology. Quist and Chapela's paper obviously represents such a challenge. That fact - not the quality of their work — together with the politics of university-industry relations, remains central to their paper's troubled reception.

The agricultural biotechnology industry undermines its own credibility by not aggressively evaluating the health and environmental implications of its products. The public will remain sceptical until it does so.

We call on scientists, Nature and other scientific journals to re-examine their commitment to agricultural biotechnology as well as their own conflicts of interest, and to actively encourage a balanced, critical evaluation of the ecological and health effects of the flow of transgenes into the environment.

For further analysis, see www.cnr. berkeley.edu/ kenw/maize/ compromised.htm.

Kenneth Worthy ${ }^{\star}$, Richard C. Strohman $\dagger$, Paul R. Billings $\ddagger$

${ }^{\star}$ Department of Environmental Science, Policy and Management, University of California, Berkeley, 135 Giannini Hall, Berkeley, California $94720-$ 3312, USA

kworthy@nature.berkeley.edu

$\dagger$ Department of Cell and Molecular Biology, University of California, Berkeley, 229 Stanley Hall, Berkeley, California 94720-3206, USA

‡GeneSage, Inc., 589 Howard Street, San Francisco, California 94105, USA

Statement of competing financial interests: the authors are recipients of educational grants from and/or employees of the University of California, Berkeley, which could lose financially from publication of this Correspondence as a result of its strategic alliance with TMRI/Syngenta.

Metz and Fütterer reply — The accusations of Worthy et al. about our Brief

Communication $^{1}$ do not relate to the scientific data of Quist and Chapela ${ }^{4}$. Our 
concern was exclusively over the quality of the scientific data and conclusions, which would have been the same whatever the motivation of the criticism.

Bad science can only undermine our understanding of nature, and the making of constructive public policy. The statement that "commercially vested interests" (that is, ties to Syngenta/ Novartis) are "central to" criticisms of the data in ref. 4 is as useless in addressing the scientific issues as would be an accusation that these data were tainted by a grudge between Chapela and his former employer (the same company). Although our connections to industry are irrelevant to the scientific issues, and hence do not warrant disclosure, we feel compelled to dispel the mischaracterization of Worthy et al.. One of us (M. M.) had TMRI funding for only one-sixth of his study at UC Berkeley, and the other's (J. F.) alleged link to TMRI relies entirely on someone else's former Berkeley association. Both of us currently have research funding exclusively from the public sector.

We are not unlike many scientists in that we have shared research and funding with industry at some point. In stating that we have "compromised positions", Worthy et al. wrongly imply that private-sector funding strips us of integrity and legitimacy in the arena of scientific discourse. Far from promoting "scientific freedom and balance", this presumption tars any scientist who can be suggested to have worked with the private sector. The only threat to academic freedom that seems to have materialized from the Berkeley/TMRI collaboration is this attitude towards scientists who might have industry links.

Matthew Metz ${ }^{*}$

Johannes Fütterer $\dagger$

${ }^{*}$ Department of Microbiology, University of Washington, Seattle, Washington 98195, USA $\dagger$ Institute of Plant Sciences, ETH, CH-8092 Zürich, Switzerland

Kaplinsky replies - I, on behalf of the authors of our Brief Communication ${ }^{2}$, state unequivocally that funding from TMRI has absolutely nothing to do with our criticisms. Worthy and co-authors are incorrect. Two of my co-authors of ref. 2 (Hake and Hay) do not receive any industry funding. Funding information for the Freeling lab (Braun, Freeling, Lisch and N. K.) is transparent and public (see http://plantbio.berkeley.edu/ freeling/lab web/fund.html); less than a quarter of it is from industry.

As Worthy et al. state, Chapela and Quist are "leading critics" of the TMRI agreement. Chapela is a board member of PANNA (http://www.panna.org/panna/ about/board.html\#ihc), an advocacy group opposing genetically modified organisms (GMOs). It is a double standard to accuse us, but not Quist and Chapela, of a conflict of interest.

Our letter was a critique of poorly conducted and interpreted science and was not pro- or anti-GMO or industry. We simply corrected what we think is bad science. Even if we were in the pockets of industry, Quist and Chapela's published results ${ }^{4}$ would still be artefactual.

Nick Kaplinsky

Department of Plant and Microbial Biology, University of California, Berkeley, California 94720, USA

1. Metz, M. \& Fütterer, J. Nature 416, 600-601 (2002).

2. Kaplinsky, N. et al. Nature 416, 601 (2002).

3. Quist, D. \& Chapela, I. H. Nature 416, 602 (2002)

4. Quist, D. \& Chapela, I. H. Nature 414, 541-543 (2001).

\section{Nature comments:}

It is highly unusual for Nature to publish a paper whose principal conclusion is shown to be not necessarily false but unsustainable on the basis of the reported evidence. The paper was not formally retracted by its authors or by Nature. In the circumstances, Nature considered it appropriate for the record to make clear to readers its revised view of its original decision to publish.

The independence of our editorial decision-making from partisan anti- or pro-technology agendas and from commercial interests is paramount in our role as a journal.

\section{Impact-factor rewards affect Spanish research}

Sir - In Spain, as in Finland ${ }^{1}$, publication of research reports in journals with a high impact factor has since 1989 officially been part of the national system for evaluating researchers' productivity. But unlike the Finnish system, the Spanish system rewards individuals rather than departments or institutions.

As stated in the Spanish parliamentary record $^{2,3}$, a bonus is awarded only for "those articles of scientific worth in journals of recognized prestige in the field. As a quality indicator, the relevance of the medium of dissemination in which each article was published shall be considered. In those disciplines for which international systems of quality of publications exist, reliance on these systems shall be obligatory."

What exactly are these "systems of quality of publication"? In mathematics and physics, chemistry, cell and molecular biology, medicine, natural science, engineering and architecture, economics and social science, the law specifies that "preference shall be given to those contributions consisting of articles in journals of recognized prestige, which shall be accepted to mean those that occupy relevant positions in the lists for science fields in the Subject Category Listings of the Journal Citation Reports of the Science Citation Index (Institute for Scientific Information [ISI], Philadelphia, PA, USA)" (ref. 4). For the first four fields, the National Commission for the Evaluation of Research Activity (CNEAI) recommends that articles should be published in journals that occupy a relevant position in the pertinent ranking, which is understood to mean the upper third of the listing ranked by impact factor. There may, however, be some flexibility under special circumstances at the discretion of the experts. The aim of this reward system is to improve the quality of Spanish science and its visibility in journals.

The consequences of this law have been first, a change in Spanish scientists' publication habits; second, an increase in the number of Spanish source items in the ISI databases ${ }^{5,6}$; and third, a levelling off of source items in Spanish bibliographic databases ${ }^{7}$. Spanish scientific productivity has doubled: more than twice as many papers were published between 1991 (immediately after the system of publication bonuses was passed into law) and 1998 compared with the period 1982-1990. The acceleration in national publication output cannot be attributed to increased financial support (research budgets had begun to shrink in real terms by 1991), international collaboration or mobility of researchers, nor can it be attributed to an overall increase in scientific activity ${ }^{8}$.

We thank K. Shashok for translating this Correspondence into English. Evaristo Jiménez-Contreras*, Emilio Delgado López-Cózar ${ }^{\star}$, Rafael Ruiz-Pérez ${ }^{\star}$, Víctor M. Fernández†

${ }^{*}$ Department of Library and Information Science, School of Library and Information Science,

University of Granada, 18071 Granada, Spain $\dagger$ Former director, National Commission for the Evaluation of Research Activity (CNEAI), Department of Biocatalysis, Institute of Catalysis, CSIC, 28049 Madrid, Spain

1. Adam, D. Nature 415, 726-729 (2002).

2. Boletín Oficial del Estado 28563 (9 September 1989).

3. Boletín Oficial del Estado 3566 (6 February 1990); Boletín Oficial del Estado 37030 (3 December 1994).

4. Boletin Oficial del Estado 35028 (20 November 1996).

5. Garfield, E. www.garfield.library.upenn.edu/papers/ mapsciworld.html.

6. Gómez, I., Bordons, M., Camí, J. Science 276, 883-884 (1997).

7. Fernández, M. T., Bordons, M., Sancho, R., Gómez, I. Arbor 639, 327-345 (1999).

8. Jiménez-Contreras, E., Moya, F., Delgado López-Cózar, E. Research Policy 2002 (in the press). 